\title{
NONLINEAR EXTENSION OF ASYMMETRIC GARCH MODEL WITHIN NEURAL NETWORK FRAMEWORK
}

\author{
Josip Arnerić $^{1}$ and Tea Poklepović ${ }^{2}$ \\ ${ }^{1}$ Faculty of Economics and Business Zagreb, \\ Department of Statistics, Trg J. F. Kennedyja 6, 1000 Zagreb, Croatia \\ jarnericeefzg.hr \\ ${ }^{2}$ Faculty of Economics Split, Department of Quantitative Methods, \\ Cvite Fiskovića 5, 21000 Split, Croatia \\ tpoklepodefst.hr
}

\begin{abstract}
The importance of volatility for all market participants has led to the development and application of various econometric models. The most popular models in modelling volatility are GARCH type models because they can account excess kurtosis and asymmetric effects of financial time series. Since standard GARCH $(1,1)$ model usually indicate high persistence in the conditional variance, the empirical researches turned to GJR-GARCH model and reveal its superiority in fitting the asymmetric heteroscedasticity in the data. In order to capture both asymmetry and nonlinearity in data, the goal of this paper is to develop a parsimonious $N N$ model as an extension to GJR-GARCH model and to determine if GJR-GARCH-NN outperforms the GJR-GARCH model.
\end{abstract}

\section{KEYWORDS}

conditional volatility, GARCH model, GJR model, Neural Networks, emerging markets

\section{INTRODUCTION}

Modelling volatility, i.e. returns fluctuations, has been a topic of interest to economic and financial researchers. Portfolio managers, option traders and market makers are all interested in volatility forecasting in order to get higher profits or less risky positions.

The most popular models in modelling volatility are generalized autoregressive conditional heteroskedasticity (GARCH) type models which can account excess kurtosis and asymmetric effects of high frequency data, time varying volatility and volatility clustering. The first autoregressive conditional heteroscedasticity model (ARCH) was proposed by Engle [1] who won a Nobel Prize in 2003 for his contribution to modelling volatility. The model was extended by Bollerslev [2] by its generalized version $(\mathrm{GARCH})$. However, standard GARCH $(1,1)$ model usually indicates high persistence in the conditional variance, which may originate from structural changes in the variance process. Hence the estimates of a GARCH model suffer from a Jan Zizka et al. (Eds) : CCSEIT, AIAP, DMDB, MoWiN, CoSIT, CRIS, SIGL, ICBB, CNSA-2016 pp. 101-111, 2016. @ CS \& IT-CSCP 2016

DOI : $10.5121 /$ csit.2016.60609 
substantial upward bias in the persistence parameters. Also, it is often difficult to predict volatility using traditional GARCH models because the series is affected by different characteristics: non-stationary behaviour, high persistence in the conditional variance, asymmetric behaviour and nonlinearity. Due to practical limitations of these models different approaches have been proposed in the literature. Some of them are developed for resolving the asymmetric behaviour problem and some of them the nonlinearity in variance. Diebold [3] found that volatility models that fail to adequately incorporate nonlinearity are subject to an upward bias in the parameter estimates which results in strong forms of persistence that occurs especially in high volatility periods in financial time series and this influences the out-of-sample forecasts of single regime type GARCH models. The empirical researches reveal that among asymmetric models, Glosten, Jaganntahn and Runkle's [4] sign-ARCH model, i.e. GJR-GARCH model outperforms all the other GARCH-type models. Moreover, to account for nonlinearity, in recent researches much attention is given to neural network models (NN) in forecasting volatility.

The NNs are a valuable tool for modelling and prediction of time series in general ([5]; [6]; [7]; [8]; [9]; [10]; [11]; [12]; [13]). Most financial time series indicate existence of nonlinear dependence, i.e. current values of a time series are nonlinearly conditioned on information set consisting of all relevant information up to and including period $t-1$ ([14]; [15]; [16]; [17]). The feed-forward neural networks (FNN), i.e. multilayer perceptron, are most popular and commonly used. They are criticized in the literature for the high number of parameters to estimate and they are sensitive to overfitting ([18]; [19]).

The objective of this paper is to develop a parsimonious NN model as an extension to GJRGARCH model which will capture the nonlinear relationship between past return innovations and conditional variance. The second objective of this paper is to determine if GJR-GARCH-NN model outperforms GJR-GARCH models when there is high persistence of the conditional variance. This paper contributes to existing literature in several ways. Firstly, this paper introduces $\mathrm{NN}$ as semiparametric approach, which combines flexibility of nonparametric methods and the interpretability of parameters of parametric methods, and attractive econometric tool for conditional volatility forecasting. NN models have continuously been observed as a nonparametric method relying on automatically chosen NN provided by various software tools, which is unjustified from the econometric perspective. Therefore, in this paper the "black box" will be opened. Secondly, in this paper new NN model is defined, as an extension to GJRGARCH models, and estimated. Although this paper relies on paper from Donaldson and Kamstra [20] it contributes to the literature by estimating an additional parameter $\lambda$ which was previously set in advance. Finally, this paper contributes to the literature of emerging market economies with the newest data.

The remainder of this paper is organized as follows. Section 2 presents the literature review. Section 3 describes the data and methodology. Section 4 presents the obtained empirical results and discussion. Finally, some conclusions and directions for future research are provided in Section 5 .

\section{LITERATURE REVIEW}

Donaldson and Kamstra [20] in their paper construct a seminonparametric nonlinear GARCH model, based on NN approach, and evaluate its ability to forecast stock return volatility on stock exchanges in London, New York, Tokyo and Toronto using daily stock returns from 1970 to 
1990. They compared this constructed NN model with performances of other most commonly used volatility models, i.e. GARCH, EGARCH and GJR-GARCH model, in in- and out-ofsample comparison and within different markets. The results reveal that GJR-GARCH model fits the asymmetric heteroscedasticity in the data better than GARCH and EGARCH models, however the best performing model of all seems to be the newly introduced NN model. The authors present the new methodology which is applied in advancing markets, however, the properties of selected methodology are not yet tested in emerging markets. Moreover, the number of hidden neurons is obtained by selecting the best alternative model in the grid $[0,5]$ parameter space using Schwarz information criterion. In this paper the number of hidden units in three-layer $\mathrm{NN}$ is specified in advance for the more suitable comparison between models. This paper contributes in estimating an additional parameter $\lambda$ which was in presented paper set in advance.

Teräsvirta et al [13] present similar methodology as [20] based on Medeiros et al [11] approach and use it as AR-NN type model showing the potential of their proposed modelling approach in two applications: sunspot series and US unemployment series. Moreover, they clearly combine the NN model so as to be able to explain it as econometric model.

Bildirici and Ersin [21] analyse the volatility of stock returns on Istanbul Stock Exchange (ISE) in period from 1987 to 2008 using daily closing prices of ISE 100 index. They compare and combine GARCH, EGARCH, GJR-GARCH, TGARCH, NGARCH, SAGARCH, PGARCH, APGARCH, NPGARCH with NN models in their forecasting abilities. The NN models are retrained with conjugate gradient descent algorithm after the training with backpropagation. They conclude that NN models improved the generalization and forecasting ability of GARCH models. In their paper the models are not properly explained from an econometric perspective, nor are the findings explained from the perspective to the real time data. Moreover, the parameters of the models are nor presented or explained.

Their later paper, Bildirici and Ersin [22], relies on paper from [20] and [21] to analyse the nonlinearity and leptokurtic distribution of stock returns on ISE in period from 1986 to 2010 and benefits from both LSTAR and NN type of nonlinearity, i.e. this paper proposes several LSTARGARCH-NN family models. GARCH, FI-GARCH, APGARCH and FIAPGARCH models are augmented with a NN model. They conclude that extended GARCH models forecast better than GARCH models; LSTAR-LST-GARCH show significant improvement in out-of-sample forecasting; MLP-GARCH models provide similar results to LSTAR-LST-GARCH models; LSTAR-LST-APGARCH-MLP model provided the best overall performance. To estimate NN models, the number of hidden neurons ranges from 3 to 10 and the best model is selected based on MSE or RMSE. Moreover, each of the selected model architecture is estimated 20 times for 8 different NN models and to obtain parsimony the appropriate model is selected based on AIC. Although there is a vast number of econometric models for modelling conditional volatility presented and estimated in this paper, along with an econometric presentation of NN models, estimation of 100 different $\mathrm{NN}$ models with hidden neurons ranging from 3 to 10 and comparing models with different specifications is econometrically unjustified.

Bildirici and Ersin [23] propose a family of nonlinear GARCH models that incorporate fractional integration (FI) and asymmetric power (AP) properties to MS-GARCH processes. Moreover, they augment the MS-GARCH type models with NN to improve forecasting accuracy. Therefore, the proposed MS-ARMA-FIGARCH, APGARCH, and FIAPGARCH processes are further augmented with MLP, RBF, Recurrent NN, and Hybrid NN type NNs. The MS-ARMA-GARCH 
family and MS-ARMA-GARCH-NN family are utilized for modelling the daily stock returns of the ISE Index. Forecast accuracy is evaluated with MAE, MSE, and RMSE error criteria and Diebold-Mariano test for predictive accuracy. They conclude that the FI and AP counterparts of MS-GARCH model provided promising results, while the best results are obtained for their NN based models. Moreover, among the models analysed, the models MS-ARMA-FIAPGARCHHNN and MS-ARMA-FIAPGARCH-RNN provided the best forecast performances over the single regime GARCH models and over the MS-GARCH model. Parameters of NN models are not explained econometrically, although $\mathrm{NN}$ are regarded as econometric model instead of nonparametric model.

Mantri et al [24] apply different methods, i.e. GARCH, EGARCH, GJR-GARCH, IGARCH and NN models for calculating the volatilities of Indian stock markets. Two networks are presented: single input (low index) single output (high index level) and multiple inputs (open, high and low index level) single output (close index level). The data from 1995 to 2008 of BSE Sensex and NSE Nifty indices are used to calculate the volatilities. The authors conclude that the MISO-NN model should be used instead of SISO-NN model and that there is no difference in the volatilities of Sensex and Nifty estimated under the GARCH, EGARCH, GJR-GARCH, IGARCH and NN models.

In their later paper Mantri et al. [25] focused on the problem of estimation of volatility of Indian Stock market. The paper begins with volatility calculation by ARCH and GARCH models of financial computation up to lag 3. The results are compared to NN model using R2. It can be concluded that $\mathrm{NN}$ can be used as a best choice for measuring the volatility of stock market. These papers provide no information about the particular NN model used, NN is not explained as econometric model, and therefore the papers are not suitable for deciding on suitability of the models.

Bektipratiwi and Irawan [26] propose an alternative forecasting model based on the combinations between RBF and EGARCH model to model stock returns of Bank Rakyat Indonesia Tbk for the period from 2003 to 2011. They use RBF to model the conditional mean and EGARCH to model the conditional volatility and propose a regression approach to estimate the weights and the parameters of EGARCH using maximum likelihood estimator. The relevant explanatory variables are chosen based on its contribution of giving greater reduction in the in-sample forecast errors. They selected 11 inputs for RBF model, and 5 hidden neurons based on trial and error procedure. Based on SIGN test, the best forecast is obtained by RBF-EGARCH model for 100 steps ahead.

All of the above researches combine GARCH-type and NN models by adding the NN structure to existing GARCH-type models in search of the suitable model for forecasting conditional variance of stock returns. The proposed methodology is empirically tested on developed markets, however not on developing capital markets of Central and Eastern Europe. Because of the uniqueness of these emerging capital markets, it is important to test features of proposed methodology in this particular segment. Moreover, some of the papers use NN as nonparametric estimation technique, neglecting the interpretability of parameters which could be obtained by using NN as econometric tool. In this paper NN will be observed as semiparametric approach combining the flexibility of nonparametric methods and the interpretability of parameters of parametric methods. Another contribution of the paper is in a priori specified structure of NN models in order to be comparable to the GARCH-type models and the estimation of additional parameter which was not estimated before. 


\section{DATA AND METHODOLOGY}

The most widespread approach to volatility modelling consists of the GARCH model of Bollerslev [2] and its numerous extensions that can account for the volatility clustering and excess kurtosis found in financial time series. The accumulated evidences from empirical researches suggest that the volatility of financial markets can be appropriately captured by standard GARCH(1,1) model ([27]) since it gives satisfactory results with small number of parameters to estimate. According to Bollerslev [2] GARCH $(1,1)$ can be defined as:

$$
\begin{aligned}
& r_{t}=\mu_{t}+\varepsilon_{t} \\
& \varepsilon_{t}=u_{t} \cdot \sqrt{\sigma_{t}^{2}} \\
& u_{t}: \text { i.i.d. }(0,1) \\
& \sigma_{t}^{2}=\alpha+\beta \cdot \varepsilon_{t-1}^{2}+\gamma \cdot \sigma_{t-1}^{2}
\end{aligned}
$$

where $\mu_{t}$ is the conditional mean of return process $\left\{r_{t}\right\}$, while $\left\{\varepsilon_{t}\right\}$ is the innovation process with its multiplicative structure of identically and independently distributed random variables $u_{t}$. The last equation in (1) is conditional variance equation with $\operatorname{GARCH}(1,1)$ specification which means that variance of returns is conditioned on the information set $I_{t-1}$ consisting of all relevant previous information up to and including period $t-1$. $\operatorname{GARCH}(1,1)$ model is covariancestationary if and only if $\beta+\gamma<1$ ([2]). In particular, $\operatorname{GARCH}(1,1)$ model usually indicates high persistence in the conditional variance, i.e. integrated behavior of the conditional variance when $\beta+\gamma=1$ (IGARCH). The reason for the excessive GARCH forecasts in volatile periods may be the well-known high persistence of individual shocks in those forecasts. Relevant researches ([28]; [29]) show that this persistence may originate from structural changes in the variance process. High volatility persistence means that a long time period is needed for shocks in volatility to die out (mean reversion period).

Although GARCH models are the most popular and widely used in empirical researches and among practitioners due to their ability of describing the volatility clustering, excess kurtosis and fat-tailedness of the data, they cannot capture the asymmetric behavior of volatility. This means that negative shocks affect volatility quite differently than positive shocks. Therefore, different asymmetric models have been developed and used in empirical researches such as EGARCH, GJR-GARCH, TARCH, PGARCH, APGARCH among the others. However, the results from Engle and Ng [30] of Japanese stock returns suggest that Glosten, Jaganntahn and Runkle's [4] sign-ARCH model, usually called GJR model, shows the most potential in outperforming the traditional GARCH model. Moreover, in recent literature it also proved to capture the asymmetric behavior in data better that the other models ([20]). Therefore, GJR-GARCH model is considered, i.e. GJR-GARCH $(1,1,1)$ is given by:

$$
\begin{aligned}
& \sigma_{t}^{2}=\alpha+\beta \cdot \varepsilon_{t-1}^{2}+\gamma \cdot \sigma_{t-1}^{2}+\phi \cdot D_{t-1} \cdot \varepsilon_{t-1}^{2} \\
& D_{t-1}=\left\{\begin{array}{lll}
1 & \text { if } & \varepsilon_{t-1}<0 \\
0 & \text { if } & \varepsilon_{t-1} \geq 0 .
\end{array}\right.
\end{aligned}
$$


As can be seen from (2), GJR-GARCH model is just an augmentation of GARCH model that allows past negative unexpected returns to affect volatility differently than positive unexpected returns. When $\phi>0$ negative shocks will have a larger impact on conditional variance. For GJRGARCH stationarity condition is satisfied if $\beta+\gamma+\phi / 2<1$.

An alternative solution to overcome the problems found for standard GARCH $(1,1)$ model is to define appropriate neural network (NN), i.e. by extending the GJR-GARCH $(1,1,1)$ model with NN model, significant improvements can be found.

The NN is an artificial intelligence method, which has recently received a great deal of attention in many fields of study. Usually NN can be seen as a nonparametric statistical procedure that uses the observed data to estimate the unknown function. A wide range of statistical and econometric models can be specified modifying the structure of the network, however NN often give better results. Empirical researches show that NN are successful in forecasting extremely volatile financial variables that are hard to predict with standard econometric methods such as: exchange rates ([7]), interest rates ([6]) and stocks ([8]). The most commonly used type of NN in empirical researches is multi-layer feed-forward neural networks (FNN).

The FNN forwards information from input layer to output layer through a number of hidden layers. Neurons in a current layer connect to neuron of the subsequent layer by weights and an activation function. In order to obtain weights backpropagation (BP) learning algorithm, which works by feeding the error back through the network, is mostly used. The weights are iteratively updated until there is no improvement in the error function. This process requires the derivative of the error function with respect to the network weights. The mean of squared error (MSE) is the conventional least square objective function in a $\mathrm{NN}$, defined as mean of squared differences between the observed and fitted values of time series. The FNN with linear component can be written as:

$$
\hat{y}_{t}=f\left(\phi_{c o}+\sum_{i=1}^{p} \phi_{i o} x_{t, i}+\sum_{h=1}^{q} \phi_{h o} g\left(\phi_{c h}+\sum_{i=1}^{p} \phi_{i h} x_{t, i}\right)\right)
$$

where $t$ is a time index, $\hat{y}_{t}$ is the output vector, $x_{t, i}$ is the input matrix with $i$ variables, $f(\cdot)$ and $g(\cdot)$ are activation functions (usually linear and logistic respectively). Index $c$ is the constant, $i$ is the input, $h$ is the hidden, and $o$ is the output neuron. $\phi_{c o}$ denotes the weight of the direct connection between the constant and output, $\phi_{i o}$ denote the weights of direct connection from inputs to output, $\phi_{c h}$ denote the weights for the connections between the constant and hidden neurons. The weights $\phi_{i h}$ and $\phi_{h o}$ denote the weights for the connections between the inputs and hidden neurons and between the hidden neurons and output. NN with $p$ inputs and $q$ outputs has the abbreviation $\mathrm{FNN}(\mathrm{p}, \mathrm{q})$.

However, the disadvantage of FNN is the problem of overfitting. It occurs due to the inclusion of multiple hidden layers or multiple neurons in hidden layer which, with existing theoretically based number of inputs (independent variables) and lagged outputs (dependent variables), increases the number of parameters to estimate. Therefore, in this paper NN are observed only as an extension to GJR-GARCH type model with the structure defined in advance to benefit from 
parsimonious model in order to avoid the problem of overfitting. The GJR-GARCH-NN $(1,1,1,1)$ as a nonlinear extension to GJR-GARCH model is defined as:

$$
\begin{aligned}
& \sigma_{t}^{2}=\alpha+\beta \cdot \varepsilon_{t-1}^{2}+\gamma \cdot \sigma_{t-1}^{2}+\phi \cdot D_{t-1} \cdot \varepsilon_{t-1}^{2}+\xi \psi\left(z_{t} \lambda\right) \\
& D_{t-1}=\left\{\begin{array}{lll}
1 & \text { if } \quad \varepsilon_{t-1}<0 \\
0 & \text { if } \quad \varepsilon_{t-1} \geq 0
\end{array}\right. \\
& \psi\left(z_{t} \lambda\right)=\frac{1}{1+e^{\lambda z_{t-1}}} \\
& z_{t-1}=\frac{\varepsilon_{t-1}-E(\varepsilon)}{\sqrt{E\left(\varepsilon^{2}\right)}} .
\end{aligned}
$$

where $\psi\left(z_{t} \lambda\right)$ specifies the logistic function in hidden unit of neural network with 1 hidden neuron, $z_{t-1}$ provides a normalization of $\varepsilon$ necessary to prepare the lagged unexpected returns as inputs into the nodes. All the data are transformed using the in-sample mean and variance. Donaldson and Kamstra [20] chose $\lambda$ in advance from a uniform random number generator so they lie between -2 and 2 in order to achieve the identification of parameters $\xi$, and then parameters $\alpha, \beta, \gamma, \phi, \xi$ are estimated with maximum likelihood. In this paper $\lambda$ are defined between -2 and 2 , however they are estimated with maximum likelihood just as other parameters.

The data set consists of returns of the daily closing prices obtained from stock exchanges in period from January 2011 until September 2014 for selected European emerging markets, i.e. Bulgaria, Croatia, Czech, Romania, Slovakia and Slovenia. Data is obtained from Thomson Reuters database.

\section{EMPIRICAL RESEARCH}

In order to investigate GARCH-type models it is important to give an overview of the sample, i.e. descriptive statistics. From Table 1 can be seen that in observed period European emerging markets have negative expected returns. The lowest risk is observed in Slovakia and Slovenia and the highest risk in Czech Republic and Romania. Each distribution shows asymmetric behavior and leptokurtosis. Moreover, time series is not stationary since the variance of returns is time varying. Detailed results are omitted due to a lack of space. They are available from authors upon request.

Parameters for GJR-GARCH $(1,1,1)$ model are estimated in SAS software using the maximum likelihood method and the results for selected markets with estimated parameters and the value of Log-Likelihood (LL) is given in Table 2. The results reveal that asymmetric behavior is statistically significant in all markets and since $\phi<0$ the positive shocks will have a larger impact on conditional variance. In developed markets this parameter is usually positive indicating the opposite conclusions. 
Table 1. Descriptive statistics of daily returns for selected markets

\begin{tabular}{|l|c|c|c|c|c|c|c|}
\hline & N & Min & Max & $\boldsymbol{\mu}$ & $\boldsymbol{\sigma}$ & $\boldsymbol{\alpha}_{\mathbf{3}}$ & $\boldsymbol{\alpha}_{\mathbf{4}}$ \\
\hline BULGARIA & 2177 & $-0,1136$ & 0,0729 & $-0,00044$ & 0,01307 & $-1,05$ & 10,39 \\
\hline CROATIA & 2177 & $-0,1076$ & 0,1477 & $-0,00024$ & 0,01273 & 0,14 & 18,43 \\
\hline CZECH & 2177 & $-0,1618$ & 0,1109 & $-0,00032$ & 0,01521 & $-0,82$ & 15,32 \\
\hline ROMANIA & 2177 & $-0,1311$ & 0,1056 & $-0,00005$ & 0,01639 & $-0,50$ & 8,64 \\
\hline SLOVAKIA & 2177 & $-0,1481$ & 0,1188 & $-0,00022$ & 0,01153 & $-1,52$ & 29,90 \\
\hline SLOVENIA & 2177 & $-0,0843$ & 0,0835 & $-0,00032$ & 0,01189 & $-0,46$ & 7,47 \\
\hline
\end{tabular}

Table 1. Parameter estimates of GJR-GARCH $(1,1,1)$ model with values of Log-Likelihood (LL)

\begin{tabular}{|r|c|c|c|c|c|c|}
\hline & BULGARIA & CROATIA & CZECH & ROMANIA & SLOVAKIA & SLOVENIA \\
\hline$\mu$ & -0.00007 & -0.00021 & -0.00008 & $0.000367 *$ & $5.36 \mathrm{E}-06$ & -0.00014 \\
\hline$\alpha$ & $7.14 \mathrm{E}-06 * * *$ & $4.56 \mathrm{E}-07 * * *$ & $4.42 \mathrm{E}-03 * * *$ & $4.27 \mathrm{E}-06 * * *$ & $0.000028 * * *$ & $0.000012 * * *$ \\
\hline$\beta$ & $0.307146 * * *$ & $0.116087 * * *$ & $0.171614 * * *$ & $0.190021 * * *$ & $0.078577 * * *$ & $0.279281 * * *$ \\
\hline$\gamma$ & $0.703246 * * *$ & $0.912962 * * *$ & $0.846355^{* * *}$ & $0.824793 * * *$ & $0.797184 * * *$ & $0.697366 * * *$ \\
\hline$\phi$ & $-0.08503 * *$ & $-0.05222 * * *$ & $-0.08193 * * *$ & $-0.04265 *$ & $-0.09016 * * *$ & $-0.14047 * * *$ \\
\hline LL & 6970.803 & 7227.855 & 6611.161 & 6470.448 & 6649.694 & 6945.997 \\
\hline
\end{tabular}

Note: Parameter estimates are significant at $1 \%(* *), 5 \%(* *)$ and $10 \%(*)$ significance level

Parameters for GJR-GARCH-NN(1,1,1,1) model are estimated in SAS software using the maximum likelihood method and the results for selected markets with estimated parameters and the value of Log-Likelihood (LL) is given in Table 3. This model has two additional parameters to estimate: $\xi$ and $\lambda$. Parameter $\xi$ is in each market positive and statistically significant. Moreover, the Log-Likelihood is in GJR-GARCH-NN model larger than in simpler model. All these findings lead to a conclusion that extending the GJR-GARCH with the NN model, i.e. adding the nonlinearity in the model, is statistically significant and improves the models' fit

Table 3. Parameter estimates of GJR-GARCH-NN $(1,1,1,1)$ model with values of Log-Likelihood (LL)

\begin{tabular}{|r|c|c|c|c|c|c|}
\hline & BULGARIA & CROATIA & CZECH & ROMANIA & SLOVAKIA & SLOVENIA \\
\hline$\mu$ & -0.00007 & -0.00018 & -0.00011 & 0.000391 & -0.0002 & -0.00015 \\
\hline$\alpha$ & $-0.03772 * * *$ & $-0.03771 * * *$ & $-0.03772 * * *$ & $-0.03761 * * *$ & $-0.03787 * * *$ & $-0.03776 * * *$ \\
\hline$\beta$ & $0.311529 * * *$ & $0.12463 * * *$ & $0.151603 * * *$ & $0.19557 * * *$ & $0.364962 * * *$ & $0.349813^{* * *}$ \\
\hline$\gamma$ & $0.703012 * * *$ & $0.911607 * * *$ & $0.848221 * * *$ & $0.830741 * * *$ & $0.6018284 * * *$ & $0.697389 * * *$ \\
\hline$\phi$ & $-0.09305 * * *$ & $-0.06524 * * *$ & -0.04615 & $-0.06322^{* * *}$ & $-0.27835 * * *$ & $-0.27628^{* * *}$ \\
\hline$\xi$ & $0.075462 * * *$ & $0.075429 * * *$ & $0.075448 * * *$ & $0.075225 * * *$ & $0.075971 * * *$ & $0.075546 * * *$ \\
\hline$\lambda$ & 0.000028 & 0.000039 & -0.00021 & 0.000137 & $0.000798 *$ & $0.000519 * * *$ \\
\hline LL & 6970.826 & 7228.358 & 6612.326 & 6470.998 & 6657.298 & 6950.291 \\
\hline
\end{tabular}

Note: Parameter estimates are significant at $1 \%(* * *), 5 \%(* *)$ and $10 \%(*)$ significance level 


\section{CONCLUSIONS}

Modelling volatility, i.e. returns fluctuations, is in the main focus of the paper. This research begins with the most widespread approach to volatility modelling, i.e. $\operatorname{GARCH}(1,1)$ model. Due to its disadvantage in capturing the asymmetric behavior $\operatorname{GJR}-\operatorname{GARCH}(1,1,1)$ model is introduced. However, both models fail to model nonlinearity in data and, therefore $\mathrm{NN}$ model as an extension to GJR-GARCH model is defined, i.e. parsimonious GJR-GARCH-NN model. This paper estimates the parameters of both simple and extended GJR-GARCH model and compares these models using data for selected European emerging markets. Moreover, NN are presented as an econometric tool. Results of this paper confirm conclusions of previous researches about superiority of NN versus other linear and nonlinear models. However, they are still a challenge for the researchers in order to improve their performances in forecasting conditional variance of stock returns and time series in general. The out-of-sample predictive performance, inclusion of more hidden neurons or other architectures, the use of different algorithms in the network training, open space for future work and further studies.

\section{ACKNOWLEDGEMENTS}

This work has been fully supported by Croatian Science Foundation under the project "Volatility measurement, modeling and forecasting" (5199).

\section{REFERENCES}

[1] Engle, R.F. (1982) "Autoregressive conditional heteroscedasticity with estimates of the variance of UK inflation”, Econometrica, Vol. 41, pp. 135-155.

[2] Bollerslev, T. (1986) “Generalized autoregressive conditional heteroscedasticity", Journal of Econometrics, Vol. 31, pp. 307-327.

[3] Diebold, F. (1986) "Comment on modelling the persistence of conditional variances," Econometric Reviews, Vol. 5, pp. 51-56.

[4] Glosten, L.R., Jagannathan, R. \& Runkle, D.E. (1993) "On the Relation between the Expected Value and the Volatility of the Nominal Excess Return on Stocks", The Journal of Finance, Vol. 48, No. 5 (Dec., 1993), pp. 1779-1801.

[5] Balkin, S.D. (1997) "Using Recurrent Neural Networks for Time Series Forecasting”, Working Paper Series number 97-11, International Symposium on Forecasting, Barbados.

[6] Täppinen, J. (1998) "Interest rate forecasting with neural networks", Government Institute for Economic Research, Vatt-Discussion Papers, 170.

[7] Dunis, C.L. \& Williams, M. (2002) "Modelling and trading the euro/US dollar exchange rate: Do neural networks perform better?", Journal of Derivatives \& Hedge Funds, Vol. 8, No. 3, pp. 211-239.

[8] Zekić-Sušac, M. and Kliček, B. (2002) “A Nonlinear Strategy of Selecting NN Architectures for Stock Return Predictions", Finance, Proceedings from the 50th Anniversary Financial Conference Svishtov, Bulgaria, 11-12 April, Svishtov, Veliko Tarnovo, Bulgaria: ABAGAR, pp. 325-355. 
[9] Tal, B. (2003) "Background Information on our Neural Network-Based System of Leading Indicators", CBIC World Markets, Economics \& Strategy

[10] Ghiassi, M., Saidane, H. \& Zimbra, D.K. (2005) "A dynamic artificial neural network model for forecasting time series events", International Journal of Forecasting, Vol. 21, pp. 341-362.

[11] Medeiros, M.C., Teräsvirta, T. \& Rech, G. (2006) "Building Neural Network Models for Time Series: A Statistical Approach”, Journal od Forecasting, No. 25, pp. 49-75.

[12] Kuan, C.-M. \& White, H. (2007) "Artificial neural networks: an econometric perspective", Econometric Reviews, Vol. 13, pp. 1-92.

[13] Teräsvirta, T., Tjøstheim, D. \& Granger, C.W.J. (2008) Modelling nonlinear economic time series, Advanced texts in econometrics, Oxford, New York, Oxford University Press

[14] Gonzales, S. (2000) "Neural Networks for Macroeconomic Forecasting: A Complementary Approach to Linear Regression Models", Working Paper 2000-07.

[15] Hwarng, H.B. (2001) "Insights into neural-network forecasting of time series corresponding to $\operatorname{ARMA}(p, q)$ structures”, Omega, Vol. 29, pp. 273-289.

[16] Zhang, G.P. (2003) "Time series forecasting using hybrid ARIMA and neural network model", Neurocomputing, Vol. 50, pp. 159-175.

[17] Aminian, F., Suarez, E.D., Aminian, M. \& Walz, D.T. (2006) "Forecasting Economic Data with Neural Networks", Computational Economics, Vol. 28, pp. 71-88.

[18] Lawrence, S., Giles, C.L. \& Tsoi, A.C. (1997) "Lessons in Neural Network Training: Overfitting May be Harder than Expected", Proceedings of the Fourteenth National Conference on Artificial Intelligence, AAAI-97, pp. 540-545.

[19] Franses, P.H. \& van Dijk, D. (2003) Nonlinear Time Series Models in Empirical Finance, Cambridge University Press.

[20] Donaldson, R.G. \& Kamstra, M. (1997) "An Artificial Neural Network - GARCH Model for International Stock Return Volatility”, Journal of Empirical Finance, Vol. 4, No. 1, pp. 17-46.

[21] Bildirici, M. \& Ersin, Ö.Ö. (2009) "Improving forecasts of GARCH family models with the artificial neural networks: An application to the daily returns in Istanbul Stock Exchange", Expert Systems with Applications, No. 36, pp. 7355-7362

[22] Bildirici, M. \& Ersin, Ö.Ö. (2012) "Nonlinear volatility models in economics: smooth transition and neural network augmented GARCH, APGARCH, FIGARCH and FIAPGARCH models", MPRA Paper No. 40330

[23] Bildirici, M. \& Ersin, Ö.Ö. (2014) "Modelling Markov Switching ARMA-GARCH Neural Networks Models and an Application to Forecasting Stock Returns", Hindawi Publishing Corporation: The Scientific World Journal, Vol. 2014, Article ID 497941, 21 pages

[24] Mantri, J.K., Gahan, P. \& Nayak, B.B. (2010) “Artificial Neural Networks - An Application to Stock Market Volatility", International Journal of Engineering Science and Technology, Vol. 2, No. 5, pp. 1451-1460. 
[25] Mantri, J.K., Mohanty, D. \&Nayak, B.B. (2012) "Design Neural Network for Stock Market Volatility: Accuracy Measurement", International Journal on Computer Technology \& Applications, Vol. 3, No. 1, pp. 242-250.

[26] Bektipratiwi, A. \& Irawan, M.I. (2011) “A RBF-EGARCH neural network model for time series forecasting”, Proceedings of "The IceMATH 2011, Topic, pp. 1-8.

[27] Visković, J., Arnerić, J. \&Rozga, A. (2014) "Volatility Switching between Two Regimes”, World Academy of Science, Engineering and Technology, International Science Index 87, International Journal of Social, Management, Economics and Business Engineering, Vol. 8, No. 3, pp. 682 - 686.

[28] Lamoureux, C. \& Lastrapes, W. (1990) "Persistence in variance, structural change, and the GARCH model”, Journal of Business and Economic Statistics, Vol. 8, pp. 225-234.

[29] Wong, C.S. \& Li, W.K. (2001) "On a mixture autoregressive conditional heteroscedastic model", Journal of American Statistical Association, Vol. 96, No. 455, pp. 982-995.

[30] Engle, R.F. \& Ng, V.K. (1993) "Measuring and Testing the Impact of News on Volatility", The Journal of Finance, Vol. 48, No. 5 (Dec., 1993), pp. 1749-1778.

\section{AUTHORS}

\section{Josip Arnerić}

Assistant Professor, PhD. University of Zagreb, Faculty of Economics and Business Zagreb, Croatia. Scientific affiliation: econometric methods and models, financial time series and volatility, VAR models and cointegration, GARCH and MGARCH models, stochastic processes and risk management. Phone: 0038512383361. Fax: 0038512332618.E-mail: jarneric@efzg.hr

\section{Tea Poklepović}

Teaching Assistant, Doctoral student. University of Split, Faculty of Economics, Split, Croatia. Scientific affiliation: statistics and econometrics in business, finance and macroeconomics, especially econometric methods and models, time series and neural networks. Phone: 0038521430761. Fax: 0038521430701. E-mail: tpoklepo@efst.hr.
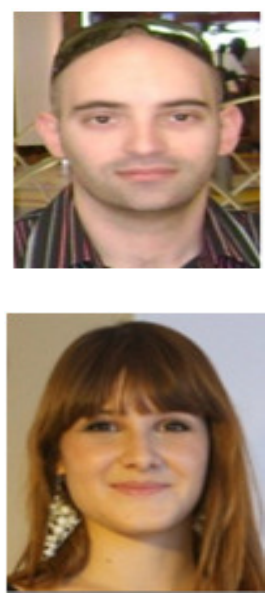